\title{
Alimentación de Characidium rachovii (Characiformes: Crenuchidae) y Pyrrhulina australis (Characiformes: Lebiasinidae) en lagunas someras de la provincia de Corrientes, Argentina
}

\author{
Mario Gabriel Ibarra Polesel ${ }^{1,2} \&$ Alicia Susana Guadalupe Poi ${ }^{1}$ \\ 1. Centro de Ecología Aplicada del Litoral- CECOAL (CCT Nordeste - CONICET- UNNE), Ruta 5 km 2.5, (3400) \\ Corrientes, Argentina; guadalupepoi@gmail.com \\ 2. Cátedra de Biología de los Artrópodos, Facultad de Ciencias Exactas y Naturales y Agrimensura, Universidad \\ Nacional del Nordeste, Avda. Libertad 5470, (3400) Corrientes, Argentina; mario.ibarrapolesel@gmail.com
}

Recibido 12-VI-2015. Corregido 05-I-2016. Aceptado 05-II-2016.

\begin{abstract}
Feeding of Characidium rachovii (Characiformes: Crenuchidae) and Pyrrhulina australis (Characiformes: Lebiasinidae) in shallow lakes of Corrientes, Argentina. Fishes display diverse feeding strategies that may undergo modifications through ontogeny or seasonally with ecological implications in the food webs. Even though the significance of fishes as top predators is recognized, the diet of many of them is still scarcely known; especially in fish that inhabit shallow lakes densely vegetated. The aim of this study was to investigate the diet of Characidium rachovii and Pyrrhulina australis of different size classes (I, II and III); during winter and summer, and estimate their trophic niche breadth. The field work was carried out in two shallow lakes fed by rain (Soto and Pampin lake, Corrientes province), from July 2011 to August 2012. The stomach contents of 104 individuals of $C$. rachovii and 91 specimens of $P$. australis were examined. Accumulation curve showed that the number of analyzed stomachs was representative for both species. Twenty three food items for $C$. rachovii and twenty eight for $P$. australis were recognized in the gut contents. According to the index of relative importance (IRI), both species feed preferably on cladocerans and midges (Chironomidae), and both species showed a large overlap in their diets (85\%), according to Morisita index. However the diversity of food items and the trophic niche breadth of $P$. australis $\left({ }^{l} D=8.86 ; B=4.76\right)$ were higher than $C$. rachovii $\left({ }^{I} D=\right.$ $3.37 ; B=2.25)$. Significant differences were observed in the diet of three sizes of $P$. australis $(\chi 2, \mathrm{p}<0.01)$ and between the sizes I and III of $C$. rachovii $(\chi 2, p=0.03)$. In both species the diversity of food items increased with increasing their size. Between seasons, the diet differences were significant for both species $(\chi 2, \mathrm{p}<0.0001)$. The high feeding overlap indicates that both species mostly use the same resources. Notwithstanding this, both the number of food items as the diversity and breadth of the trophic niche, suggest that these species use different feeding strategies, $C$. rachovii showed a trend towards specialization in microcrustacean consumption; while $P$. australis exhibited a broader trophic spectrum, incorporating insects from littoral areas and from the terrestrial community. The loss of the trophic dominant resource, by increasing urbanization on the margin of the studied shallow lakes, could lead to increased competition, due to the wide diet overlap in both species. Rev. Biol. Trop. 64 (2): 603-615. Epub 2016 June 01.
\end{abstract}

Key words: fish, diet, trophic niche, peri-urban lakes, Argentine Northeast.

En el sector noroccidental de la provincia de Corrientes (Argentina), el paisaje presenta numerosas lagunas pequeñas (de 1 a 500 ha), sub-redondeadas, localizadas sobre lomas arenosas (Poi \& Galassi, 2013) que presentan extensa cobertura de plantas acuáticas sumergidas, rodeadas de vegetación palustre. La mayoría de los antecedentes referidos a la fauna íctica de estas lagunas data de 1975 y están referidos a las lagunas de la cuenca del río Riachuelo (Bonetto et al., 1978a; Bonetto, Roldán, \& Oliver, 1978b). La mayor parte de las 129 especies de peces citadas para esta cuenca (Lopez, Miquelarena, \& Ponte Gómez, 2005), son especies de pequeño a mediano tamaño, pertenecientes a los Characiformes que viven 
entre la vegetación acuática. Estudios más recientes, fueron realizados en los esteros del Iberá (Casciotta, Almirón, \& Bechara, 2005) área que, con el triángulo nordeste de la provincia, conforman la macroregión del Iberá (Neiff, 2004).

El estudio de la alimentación de los peces es un tema de gran importancia para conocer las interacciones depredador-presa y el efecto directo o indirecto sobre las redes tróficas. $\mathrm{Si}$ bien se conoce la importancia de éstos como depredadores tope en los ambientes acuáticos, los trabajos referidos a la dieta de muchos peces son escasos, principalmente de aquellos que habitan las lagunas someras densamente vegetadas. Las áreas vegetadas proveen a los peces alimento, refugio, territorio de cortejo, sitio de desove y protección de la acción de los vientos (Welcomme, 1992). La mayor parte de la información disponible acerca de la dieta de los peces tropicales y subtropicales de agua dulce, está centrada en los ambientes lóticos (Winemiller, Agostinho, \& Caramaschi, 2008), especialmente en los grandes ríos y su planicie de inundación, donde habitan las especies de interés comercial explotadas por el hombre (Welcomme, 1992). En estos ambientes, las variaciones estacionales están relacionadas con los periodos secos y húmedos en los cuales se producen los mayores cambios en la oferta de alimento (Abelha, Agostinho, \& Goulart, 2001; Machado-Allison, 2005).

De acuerdo a Bonetto et al. (1978b), Characidium rachovii Regan, 1913 integraría el grupo de los micro-mesoanimalívoros mientras que Pyrrhulina australis Eigenmann y Kennedy, 1903 se alimentaría preferentemente de macrófitos. Estas especies son consumidas por Hoplias malabaricus (Bloch, 1794), mientras que $C$. rachovii fue hallada en la dieta de Crenicichla lepidota Heckel, 1840, lo que sugiere que ambas especies cumplen un rol clave en el funcionamiento de las lagunas someras de Corrientes.

Casciotta et al. (2005), Teixeira de Mello, González-Bergonzoni y Loureiro (2011) y Bastos, Miranda y Garcia (2013) reportan que la dieta de C. rachovii se compone de invertebrados, mientras que Fernández, Ferriz, Bentos y López (2012) le asignan la categoría de omnívoro. Estudios realizados en especies congéneres (Barreto \& Aranha, 2006; Cetra, Rodrigues-Rondineli, \& Pereira-Souza, 2011; Mazzoni, Marques, Rezende, \& Iglesias-Rios, 2012) sugieren una alimentación mayormente insectívora para el género. En lo que respecta a P. australis, su dieta es descrita brevemente por Casciotta et al. (2005), y abordada con mayor profundidad por Meschiatti y Arcifa (2002) y Arias y Rossi (2005), los cuales coinciden que los microcrustáceos e insectos son las principales presas de esta especie.

La amplitud del nicho trófico, se refiere a la flexibilidad que poseen las especies para utilizar el alimento que está a su disposición (Piet, Ret, Guruge, Vijuever, \& Van Densen, 1999), el cual está determinado en parte, por las características morfológicas del pez, su estado de desarrollo y la disponibilidad de recursos en el ambiente a lo largo del tiempo (Fernández et al., 2012). La separación trófica, ha sido señalada como el mecanismo más importante para la repartición de los recursos en las colectividades de peces (Ross, 1986). La plasticidad de la dieta dentro de una misma especie, está relacionada frecuentemente con el desarrollo ontogénico de los individuos, tanto en los ambientes de agua dulce (Abelha et al., 2001) como en los marinos (Costalago, Navarro, Álvarez-Calleja, $\&$ Palomera, 2012). En la mayoría de las especies se ha observado el consumo de plancton en algún estado de desarrollo (Welcomme, 1992), aun en especies piscívoras, los juveniles adoptan una dieta basada en microcrustáceos e insectos (Winemiller, 1989).

El objetivo de este trabajo fue a) conocer la dieta de C. rachovii y P. australis en lagunas someras periurbanas, b) cuantificar los cambios entre categorías de tallas y la variación estacional en la dieta y c) medir la amplitud del nicho trófico y el grado de solapamiento de las dietas de ambas especies. Nuestras hipótesis son 1) La dieta de los peces es más variada en los ejemplares de tallas mayores, debido a un mayor desarrollo y tamaño de estructuras 
asociadas a la alimentación (boca, musculatura), que les permite aprovechar una mayor gama de recursos. 2) La dieta de los peces es más diversa en verano, debido a la mayor actividad de insectos y otros invertebrados en esta estación 3) Los nichos tróficos de las dos especies no son similares, ya que ambas explotan recursos diferentes.

\section{MATERIALES Y MÉTODOS}

Descripción del área de estudio: Las dos lagunas estudiadas son someras, alimentadas por las lluvias locales y no conectadas a la dinámica de ríos. Estos cuerpos de agua constituyen sistemas relativamente cerrados de flujo vertical, en los que fue posible estudiar correlaciones entre la alimentación de peces y los sustratos alimenticios disponibles.

La laguna Pampín $\left(27^{\circ} 28^{\prime} 50^{\prime}\right.$ S $\left.58^{\circ} 44^{\prime} 51^{\prime \prime} \mathrm{W}\right)$ pertenece a la cuenca del río Riachuelo (Fig. 1) y recibe el aporte de efluentes domésticos de los asentamientos precarios instalados en sus márgenes. Tiene una superficie de $0.77 \mathrm{~km}^{2}$ y su profundidad varía entre 0.6 y $1.8 \mathrm{~m}$. Las plantas acuáticas dominantes (Egeria najas Planch, Eichhornia azurea (Sw.) Kunth, Hydrocleys nymphoides Willd. Buchenau y Nymphaea prolifera Wiersema) cubren 85 $\%$ de la superficie.
La laguna Soto $\left(28^{\circ} 15^{\prime} 10^{\prime \prime} \mathrm{S}-58^{\circ} 36^{\prime} 51^{\prime \prime}\right.$ W), pertenece a la cuenca del río Santa Lucía y en sus márgenes se localiza la ciudad de Saladas cuya población es de 24000 habitantes. $\mathrm{Su}$ superficie es de $0.73 \mathrm{~km}^{2}$ y la vegetación está compuesta por juncales de Schoenoplectus californicus (C.A. Mey.), Paspalidium paludivagum (Hitchc. \& Chase.), Salvinia biloba Raddi, Cabomba caroliniana A. Gray, Egeria najas Planch. y Ceratophyllum demersum L. Durante el período de estudio, la profundidad varió entre 1.4 y $1.8 \mathrm{~m}$.

El clima de la región es subtropical húmedo con inviernos suaves y libres de heladas en su mayor parte (Bruniard, 1999). De acuerdo a datos del Instituto Nacional de Tecnología Agropecuaria (INTA) Estación Experimental "El Sombrero", basados en promedios históricos, el período lluvioso ocurre en primaveraverano, con precipitaciones que oscilan entre 1300 y $1500 \mathrm{~mm}$ anuales. La temperatura presenta medias máximas comprendidas entre 27.1 y $28{ }^{\circ} \mathrm{C}$ y medias mínimas entre 16 y $17^{\circ} \mathrm{C}$. Las máximas absolutas se registran en enero y varían entre 42 y $44{ }^{\circ} \mathrm{C}$ (INTA, 2009).

En ambas lagunas el agua fue transparente (Secchi, 0.84-1.8 m) y la salinidad baja (entre 57 y $73 \mu \mathrm{S} / \mathrm{cm})$. Durante los muestreos, la temperatura del agua varió entre 13.1 y 15.3 ${ }^{\circ} \mathrm{C}$ (invierno) y entre 24 y $30.1{ }^{\circ} \mathrm{C}$ (verano).

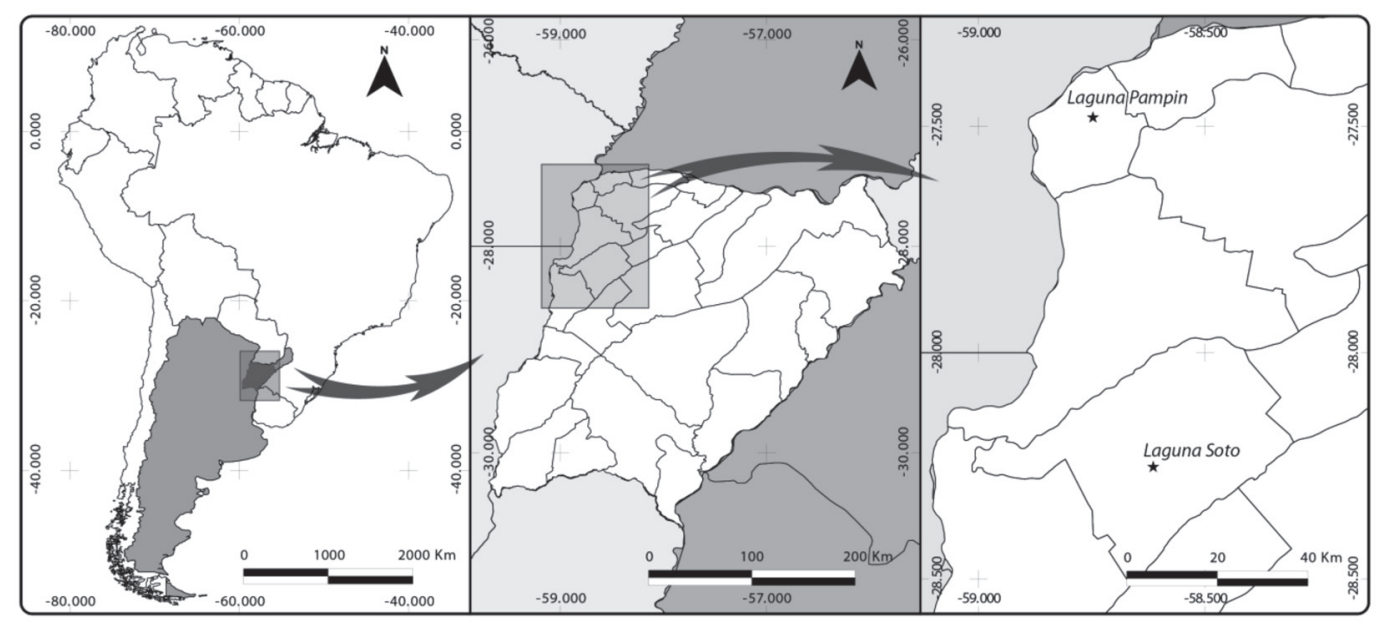

Fig. 1. Lagunas Soto y Pampín, Corrientes, Argentina. / Fig. 1. The Soto and Pampín lakes in Corrientes, Argentina. 
En la laguna Soto el $\mathrm{pH}$ fue variable (7.68.86) y hubo buena disponibilidad de oxígeno disuelto (entre 5.6 y $12.9 \mathrm{mg} / \mathrm{L}$ ), mientras que en la laguna Pampín, la concentración de oxígeno disuelto fue comparativamente menor $(3.4 \pm 1.55 \mathrm{mg} / \mathrm{L})$ y el $\mathrm{pH}$ estuvo cercano al punto neutro.

Recolecta de muestras: Las recolectas se realizaron en verano (diciembre 2011, febrero y marzo 2012) e invierno (junio y julio 2011, y julio y agosto 2012). Las capturas se hicieron entre las 9:00 y las 12:00 horas desde una embarcación en el área vegetada de ambas lagunas, utilizando un copo circular de $60 \mathrm{~cm}$ de diámetro con una apertura de malla de 1.5 $\mathrm{mm}$. Las especies objeto de este estudio fueron separadas de la vegetación y el detrito, e inmediatamente depositadas en frascos con formaldehído al $4 \%$, para finalmente ser conservadas en alcohol etílico al $70 \%$.

Actividades de laboratorio: Se registró la longitud total y estándar de cada ejemplar mediante el uso de calibre de precisión $(0.1 \mathrm{~mm})$. Para el estudio del espectro trófico, se examinaron los estómagos de 104 individuos de C. rachovii y de 91 ejemplares de $P$. australis. $\mathrm{Su}$ contenido fue vertido en portaobjetos para la observación bajo microscopio estereoscópico y microscopio binocular. Se cuantificaron e identificaron los ítem-presa mediante la utilización de claves taxonómicas (Fernández \& Domínguez, 2009; Lopretto \& Tell, 1995).

Los ítems presa fueron estudiados ordinalmente, ya que en este nivel jerárquico la mayoría de las presas pudieron ser clasificadas independientemente del grado de digestión. Para determinar si el número de estómagos examinados fue representativo, se confeccionaron curvas de acumulación de ítem-presa con base en el número de estómagos analizados en cada especie. Este análisis se realizó con el programa Estimates 8.0 (Colwell, 2006), con intervalos de confianza del $95 \%$.

Se calculó la importancia relativa de cada una de las presas utilizando el índice IRI (índice importancia relativa): IRI $=100 * \mathrm{AL} / \sum \mathrm{AL}$, donde $\mathrm{AL}=\%$ de frecuencia de la presa $+\%$ de numerosidad de la presa $+\%$ de volumen de la presa (George \& Hadley, 1979). El volumen de cada ítem alimenticio se estimó usando la fórmula del esferoide de Dunham (1983) definida como: $\mathrm{V}=4 / 3 \Pi(1 / 2 \mathrm{~L})(1 / 2 \mathrm{~A})^{2}$, donde $\mathrm{L}=$ longitud máxima de la presa y $\mathrm{A}=$ ancho máximo, y la fórmula del cilindro: $\mathrm{V}=\Pi \mathrm{r}^{2} \mathrm{~h}$. Los ítem-presa mejor conservados, fueron fotografiados con una cámara Canon Eos Rebel T3i montada a un microscopio estereoscópico Leica EZ4, con papel milimetrado de fondo. Luego, las imágenes de las presas se midieron con el programa TpsDig, versión 2.17, con una precisión de $0.01 \mathrm{~mm}$. El volumen de cada ítem, se estimó a partir de la talla promedio de varias muestras de cada ítem.

Con el fin de establecer una jerarquía para cada ítem-presa, se aplicó al IRI un criterio de categorización, el cual toma el valor más alto del índice y con base en éste, calcula el porcentaje de todos los demás valores. De esta forma los ítems se cualificaron como: fundamentales (75-100\%); secundarios (50-75\%); accesorios $(25-50 \%)$ y accidentales si fueron menores al $25 \%$ (Montori, 1991).

Para comprobar si existe variación en la composición de la dieta en relación a la talla y las estaciones climáticas, se utilizó la prueba de Chi cuadrado ( $\left.\chi^{2}\right)$. Para cada especie se fijaron tres clases de talla a partir del valor medio de la longitud estándar y los valores máximos y mínimos de los ejemplares. Los individuos que presentaron las tallas menores se los calificó como talla I (C. rachovii de $13-20 \mathrm{~mm}$ y $P$. australis de $15-23 \mathrm{~mm}$ ); aquellos cuyo tamaño fue cercano a la media se los incluyó en la talla II (21-24 y 24-29 mm respectivamente) y los de mayor longitud fueron incluidos en la talla III (25-31 y 30-40 $\mathrm{mm}$ respectivamente).

La diversidad de presas se evaluó mediante el uso de números efectivos propuesto por Jost (2006). Para este análisis se utilizó la diversidad de orden $1\left({ }^{l} D\right)$, que es el exponencial de la entropía del índice de Shannon. Se calculó mediante el índice de Levins $(B)$ la amplitud del nicho trófico para cada especie (Levins, 1968). A partir de este índice se calculó la 
amplitud estandarizada del nicho trófico $(B S)$ de Hurlbert (1978). Los valores de este índice fluctúan de 0 a 1 , mayores a 0.6 corresponden a dietas de depredadores generalistas y menores a 0.6 indican un depredador especialista.

Para conocer la superposición intra e interespecífica de las dietas se utilizó el índice simplificado de Morisita ( $\mathrm{C} \lambda$ ) propuesto por Horn (1966). Sus valores varían entre 0 (sin solapamiento) a 1 (solapamiento total).

\section{RESULTADOS}

Dieta: Se registró el contenido estomacal en el $95.2 \%(n=99 / 104)$ de los individuos de C. rachovii, y en el $96.7 \%(\mathrm{n}=88 / 91)$ de los ejemplares de P. australis. Las tallas de la primera especie variaron entre 13 y $31 \mathrm{~mm}$ de longitud estándar $($ Media $=21.9 \pm 4.1 \mathrm{~mm})$, mientras que para la segunda fue de 15 a 40 $\mathrm{mm}($ Media $=26.1 \pm 5.4 \mathrm{~mm})$.

La dieta de $C$. rachovii estuvo constituida por 23 ítems (Cuadro 1), de los cuales cladóceros, copépodos, dípteros y ostrácodos fueron los que presentaron el valor más alto de IRI. El número de presas por ejemplar varió entre
3 a 271 (Media $=21.5 \pm 36.4)$. De acuerdo a la jerarquización de la dieta, los cladóceros fueron categorizados como fundamentales, los copépodos como secundarios y dípteros y ostrácodos como accesorios.

En $P$. australis se encontraron 28 ítems siendo los cladóceros y dípteros (en su mayoría quironómidos) los más abundantes y frecuentes (Cuadro 1). El número de presas por individuo varió de 1 a 134 (Media = $11.9 \pm 19.4)$. En cuanto a la jerarquización de la dieta, los cladóceros y dípteros se identificaron como fundamentales, mientras que los rotíferos, ácaros, hemípteros (Aphididae) e insectos no identificados se cualificaron como accesorios.

Las curvas de acumulación indicaron que el número de estómagos analizados fue representativo para las dos especies, y tendieron a hacerse asintóticas a partir del estómago 99 de C. rachovii y del 87 en $P$. australis (Fig. 2). Los valores en el número de ítem-presa mostraron diferencias significativas en la dieta de las especies, ya que los intervalos de confianza (95 $\%$ ) para ambas curvas no se superponen.

Al comparar la dieta de ambas especies, se observó que tanto la diversidad de presas como

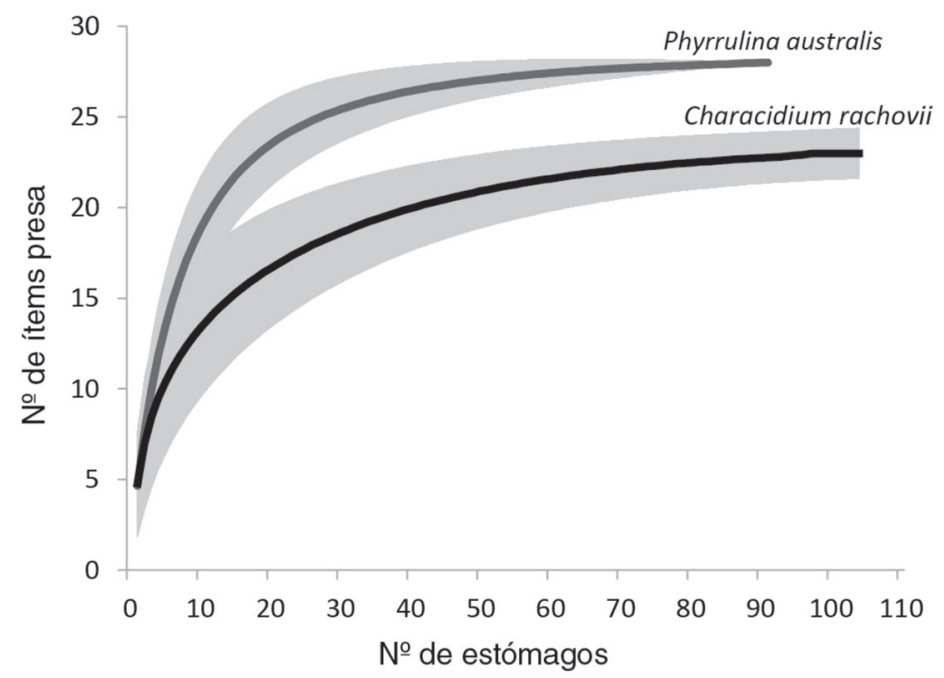

Fig. 2. Curva de acumulación de ítem-presa para los peces C. rachovii y P. australis en lagunas someras de Corrientes, Argentina. Los valores esperados en el número de presas están en líneas negras y los intervalos de confianza (95\%) sombreados en gris.

Fig. 2. Accumulation curve for $C$. rachovii and $P$. australis prey items in shallow lakes of Corrientes, Argentina. The expected values for the prey number are in dark lines and confidence intervals (95\%) gray shady. 
CUADRO 1

Espectro trófico de C. rachovii y P. australis en lagunas someras de Corrientes, Argentina

TABLE 1

Trophic spectrum of C. rachovii and P. australis in shallow lakes of Corrientes, Argentina

\begin{tabular}{|c|c|c|c|c|c|c|c|c|c|c|}
\hline \multirow{2}{*}{ Ítems } & \multicolumn{5}{|c|}{ Characidium rachovii } & \multicolumn{5}{|c|}{ Pyrrhulina australis } \\
\hline & $\% \mathrm{~N}$ & $\% \mathrm{~F}$ & $\% \mathrm{~V}$ & IRI & JD & $\% \mathrm{~N}$ & $\% \mathrm{~F}$ & $\% \mathrm{~V}$ & IRI & JD \\
\hline \multicolumn{11}{|l|}{ Arachnida } \\
\hline Acarina & 0.95 & 17.31 & 0.88 & 3.52 & 13.1 & 4.13 & 24.18 & 0.96 & 5.23 & 26.13 \\
\hline Araneae & - & - & - & - & - & 0.49 & 7.69 & 17.68 & 4.64 & 23.21 \\
\hline Pseudoescorpionida & - & - & - & - & - & 0.06 & 1.1 & 10.92 & 2.17 & 10.85 \\
\hline \multicolumn{11}{|l|}{ Chlorophyta } \\
\hline Algas filamentosas & 0.06 & 0.96 & 0.45 & 0.27 & 1.01 & 4.32 & 17.58 & 0.49 & 4. & 19.99 \\
\hline \multicolumn{11}{|l|}{ Crustacea } \\
\hline Amphipoda & 0.21 & 4.81 & 1.01 & 1.11 & 4.13 & - & - & - & - & - \\
\hline Cladocera & 62.73 & 82.69 & 0.67 & 26.84 & 100 & 40.96 & 70.33 & 0.73 & 20.02 & 100. \\
\hline Copepoda & 21.28 & 78.85 & 0.87 & 18.56 & 69.13 & 2.22 & 17.58 & 0.94 & 3.71 & 18.53 \\
\hline Decapoda & 0.06 & 0.96 & 21.29 & 4.1 & 15.27 & - & - & - & - & - \\
\hline Ostracoda & 4.88 & 42.31 & 0.77 & 8.81 & 32.83 & 1.67 & 14.29 & 0.27 & 2.9 & 14.48 \\
\hline \multicolumn{11}{|l|}{ Gastropoda } \\
\hline Pulmonata & 0.09 & 1.92 & 10.16 & 2.24 & 8.33 & - & - & - & - & - \\
\hline \multicolumn{11}{|l|}{ Insecta } \\
\hline Coleoptera & 0.12 & 3.85 & 8.45 & 2.28 & 8.5 & 0.74 & 12.09 & 9.17 & 3.94 & 19.69 \\
\hline Diptera & 4.79 & 49.04 & 0.92 & 10.06 & 37.47 & 17.33 & 70.33 & 1. & 15.84 & 79.15 \\
\hline Ephemeroptera & 0.31 & 7.69 & 12.64 & 3.79 & 14.13 & 0.31 & 5.49 & 13.71 & 3.5 & 17.51 \\
\hline Hemiptera & 0.03 & 0.96 & 0.46 & 0.27 & 1. & 4.38 & 26.37 & 0.56 & 5.6 & 27.96 \\
\hline Homoptera & 0.09 & 2.88 & 9.45 & 2.28 & 8.5 & 0.68 & 3.3 & 10.25 & 2.56 & 12.77 \\
\hline Hymenoptera & 0.03 & 0.96 & 5.17 & 1.13 & 4.22 & 0.93 & 12.09 & 5.61 & 3.33 & 16.66 \\
\hline Lepidoptera & 0.03 & 0.96 & 14.3 & 2.81 & 10.47 & 0.25 & 3.3 & 15.53 & 3.43 & 17.12 \\
\hline Odonata & 0.09 & 2.88 & 8.66 & 2.14 & 7.97 & 0.19 & 3.3 & 9.4 & 2.31 & 11.56 \\
\hline Ooteca & - & - & - & - & - & 0.06 & 1.1 & 0. & 0.21 & 1.04 \\
\hline Trichoptera & 0.21 & 5.77 & 0.53 & 1.2 & 4.46 & 0.12 & 2.2 & 0.58 & 0.52 & 2.59 \\
\hline Insecta NI & 0.12 & 1.92 & 1.62 & 0.67 & 2.51 & 5.49 & 21.98 & 0.67 & 5.03 & 25.12 \\
\hline \multicolumn{11}{|l|}{ Collembola } \\
\hline Collembola NI & - & - & - & - & - & 0.62 & 8.79 & 0.18 & 1.71 & 8.56 \\
\hline \multicolumn{11}{|l|}{ Nematoda } \\
\hline Nematoda NI & 0.89 & 14.42 & 0.6 & 2.92 & 10.89 & 1.54 & 17.58 & 0.65 & 3.53 & 17.66 \\
\hline \multicolumn{11}{|l|}{ Rotifera } \\
\hline Ploima & 2.76 & 19.23 & 0. & 4.04 & 15.06 & 5.06 & 27.47 & 0. & 5.81 & 29.04 \\
\hline \multicolumn{11}{|l|}{ Tecameba } \\
\hline Arcellida & - & - & - & - & - & 2.59 & 12.09 & 0. & 2.62 & 13.1 \\
\hline \multicolumn{11}{|l|}{ Macrófitas } \\
\hline Restos & 0.06 & 1.92 & 0.2 & 0.4 & 1.49 & 0.99 & 9.89 & 0.17 & 1.8 & 9.88 \\
\hline Semillas & 0.06 & 0.96 & 0.45 & 0.27 & 1.01 & 0.68 & 7.69 & 0.49 & 1.58 & 7.91 \\
\hline \multicolumn{11}{|l|}{ Osteichthyes } \\
\hline Restos Characiformes & - & - & - & - & - & 0.12 & 1.1 & 20.51 & 3.91 & 19.53 \\
\hline \multicolumn{11}{|l|}{ Otros } \\
\hline Arena & - & - & - & - & - & 0.19 & 3.3 & 0.03 & 0.63 & 3.14 \\
\hline Grasa & - & - & - & - & - & 0.62 & 1.1 & 3.74 & 0.98 & 4.89 \\
\hline Huevos & 0.12 & 0.96 & 0.45 & 0.28 & 1.05 & 3.27 & 9.89 & 0.49 & 2.44 & 12.19 \\
\hline Total & 100 & & 100. & 100. & & 100 & & 100. & 100. & \\
\hline
\end{tabular}

Valores porcentuales numéricos $(\% \mathrm{~N})$, frecuencia de aparición $(\% \mathrm{~F})$, volumen $(\% \mathrm{~V})$, índice de importancia relativa (IRI) y jerarquización de la dieta (JD).

Numerical percentage values $(\% \mathrm{~N})$, frequency of occurrence $(\% \mathrm{~F})$, volume $(\% \mathrm{~V})$, Relative Importance Index (IRI) and hierarchy of diet (JD). 
la amplitud del nicho fue mayor en $P$. australis $\left({ }^{1} D=8.86 ; B=4.35 ; B S=0.13\right)$ que $C$. rachovii $\left({ }^{1} D=3.37 ; B=2.25 ; B s=0.06\right)$. El índice de solapamiento trófico de Morisita indicó una superposición de la dieta entre $C$. rachovii y $P$. australis del $85 \%$.

Análisis de la dieta por tallas: En $C$. rachovii no se observaron cambios en la alimentación en relación al desarrollo de los individuos estudiados (Fig. 3). No obstante, en los ejemplares de talla III se observó un ligero incremento tanto en la diversidad de presas como en la amplitud del nicho (Cuadro 2), que estuvo acompañado por una leve reducción del número de presas registradas por ejemplar. Esto se debió a una disminución en el consumo de cladóceros y al aumento de la ingesta de otras presas. El índice de Morisita indicó un solapamiento casi total $(\mathrm{C} \lambda=0.99)$ entre las dietas de los individuos de tallas I y II y los de II y III, siendo menor entre los de talla I y III $(C \lambda=0.97)$. La prueba de $\chi 2$ demostró que solo existen diferencias alimentarias entre los individuos de las tallas I y III ( $\mathrm{p}=0.03)$.

La dieta de $P$. australis (Fig. 3) difirió en las tres tallas, siendo esta más significativa entre las tallas I y III $(\chi 2, \mathrm{p}<0.001)$ que entre las II y III $\left(\chi^{2}, \mathrm{p}=0.006\right)$ y las I y II $\left(\chi^{2}, \mathrm{p}\right.$ $=0.01)$. El número de presas registradas por individuo fue menor en los ejemplares de talla I (Cuadro 2) cuya dieta fue la menos diversa $\left({ }^{1} D=5.72\right)$. La amplitud del nicho trófico fue superior en los individuos de talla III respecto de los de talla I y II. El mayor solapamiento de dietas se registró en individuos de tallas I y II $(\mathrm{C} \lambda=0.97)$, seguido por II y III $(\mathrm{C} \lambda=$ $0.74)$ y el menor en los de I y III $(C \lambda=0.65)$. Una relación inversa, se presentó en el número medio de presas por individuo, el cual se

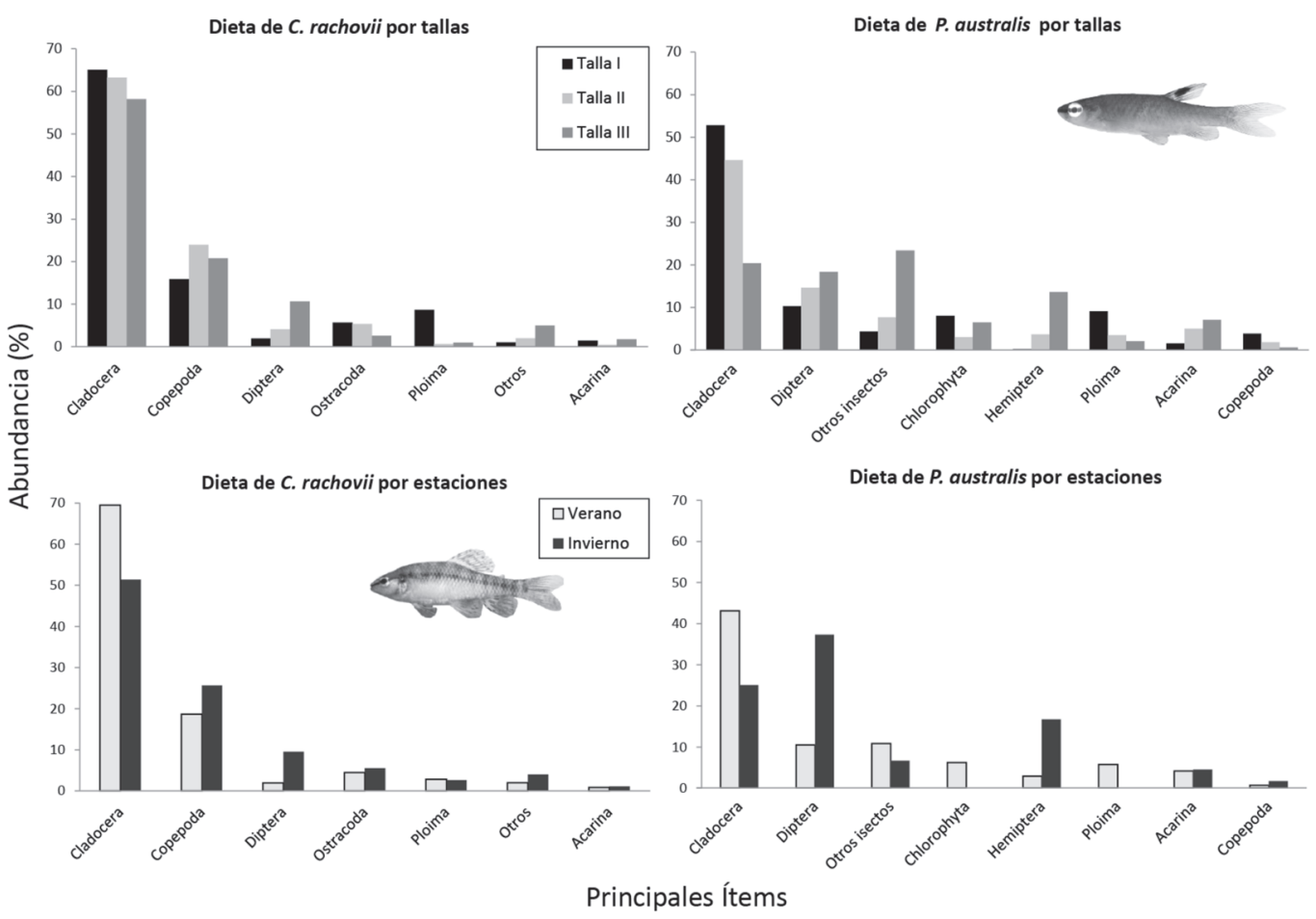

Fig. 3. Abundancia de los principales ítem-presa consumidos por C. rachovii y P. australis según sus rangos de tallas durante invierno y verano, en lagunas someras de Corrientes, Argentina.

Fig. 3. Abundance of the main prey item consumed by $C$. rachovii and $P$. australis, according to their size ranges during winter and summer, at shallow lakes of Corrientes, Argentina. 
CUADRO 2

Cambios en la dieta de C. rachovii y P. australis de acuerdo a las tallas (I, II y III) durante el invierno (Inv) y verano (Ver) en lagunas someras de Corrientes, Argentina

TABLE 2

Changes in the diet of C. rachovii y P. australis according the different sizes (I, II and III) during winter (Inv) and summer (Ver) at shallow lakes of Corrientes, Argentina

\begin{tabular}{|c|c|c|c|c|c|c|c|c|c|c|}
\hline \multirow{3}{*}{ Categoría } & \multicolumn{5}{|c|}{ Characidium rachovii } & \multicolumn{5}{|c|}{ Pyrrhulina australis } \\
\hline & \multicolumn{3}{|c|}{ Tallas } & \multicolumn{2}{|c|}{ Estaciones } & \multicolumn{3}{|c|}{ Tallas } & \multicolumn{2}{|c|}{ Estaciones } \\
\hline & I & II & III & Ver & Inv & I & II & III & Ver & Inv \\
\hline $\mathrm{N}$ & 27 & 50 & 27 & 49 & 55 & 24 & 33 & 34 & 65 & 26 \\
\hline R. L. Std. (mm) & $13-20$ & $21-24$ & $25-31$ & $13-26$ & $16-31$ & $15-23$ & $24-29$ & $30-40$ & $15-40$ & $19-38$ \\
\hline L. Std. M. (mm) & 16.9 & 22.6 & 27.1 & 19.9 & 23.9 & 20.4 & 26.2 & 32.6 & 25.9 & 28.5 \\
\hline $\mathrm{N}^{\mathrm{o}}$ items & 10 & 16 & 17 & 15 & 19 & 20 & 26 & 22 & 28 & 16 \\
\hline${ }^{1} \mathrm{D}$ & 3.17 & 3.02 & 3.88 & 2.77 & 4.27 & 5.72 & 8.39 & 11.02 & 8.71 & 5.77 \\
\hline Levins $(B)$ & 2.17 & 2.16 & 2.54 & 1.92 & 2.91 & 3.60 & 4.30 & 7.98 & 4.44 & 4.05 \\
\hline Levins Std $(B s)$ & 0.05 & 0.05 & 0.07 & 0.04 & 0.09 & 0.10 & 0.13 & 0.27 & 0.13 & 0.11 \\
\hline $\mathrm{N}^{\circ}$ M. P. & $\begin{array}{c}24.1 \\
\pm 22.1\end{array}$ & $\begin{array}{c}21.6 \\
\pm 37.4\end{array}$ & $\begin{array}{c}18.9 \\
\pm 20.0\end{array}$ & $\begin{array}{c}25.27 \\
\pm 46.77\end{array}$ & $\begin{array}{c}14.07 \\
\pm 21.62\end{array}$ & $\begin{array}{c}28.9 \\
\pm 20.5\end{array}$ & $\begin{array}{c}15.5 \\
\pm 14.3\end{array}$ & $\begin{array}{c}9.2 \\
\pm 7.3\end{array}$ & $\begin{array}{c}14.60 \\
\pm 12.33\end{array}$ & $\begin{array}{c}5.05 \\
\pm 5.38\end{array}$ \\
\hline
\end{tabular}

Número de individuos analizados (N), rango de la longitud estándar (R.L.Std.), longitud estándar media (L.Std.M.), diversidad de orden $1\left({ }^{I} D\right)$, número medio de presas por individuo $\left(\mathrm{N}^{\mathrm{o}} \mathrm{M}\right.$. P.) y desviación estándar.

Number of individuals analyzed (N), standard length range (R.L.Std.), mean standard length (L.Std.M.), diversity of order $1\left({ }^{I} D\right)$, average number of prey per individual ( ${ }^{\circ}$.M.P.) and standard deviation.

redujo de tallas grandes a chicas, siendo en los peces de talla I tres veces superior en relación a los de talla III. Este hecho estuvo asociado a un menor consumo de microcrustáceos en los ejemplares de talla II y al aumento de la ingesta de insectos acuáticos. Los individuos de talla III ingirieron además insectos de la comunidad terrestre que fueron las presas que registraron el mayor volumen.

Al analizar la superposición de la dieta entre ejemplares de $C$. rachovii y $P$. australis de la misma talla, el índice de Morisita indicó que esta fue alta en individuos de talla I ( $\mathrm{C} \lambda=$ $0.93)$, disminuyendo en los ejemplares de talla II $(\mathrm{C} \lambda=0.85)$ y talla III $(\mathrm{C} \lambda=0.54)$. Las diferencias en la dieta entre ambas especies fueron altamente significativas $(\chi 2, p<0.0001)$ para los ejemplares de las tres tallas (I, II y III).

Análisis de la dieta por estaciones: En ambas especies, la talla media de los ejemplares capturados en verano fue menor que la de los recolectados en invierno (Cuadro 2). Las diferencias estacionales en la dieta de $C$. rachovii y $P$. australis, fueron altamente significativas para ambas especies $(\chi 2, p<0.0001)$. No obstante, en la dieta de C. rachovii, los cladóceros fueron el ítem más consumido en ambas estaciones (Fig. 3). En invierno la proporción de cladóceros disminuyó y aumentó la de copépodos y quironómidos. Al analizar la composición del ítem dominante, se observó que en verano, la dieta estuvo compuesta por siete familias de cladóceros (Macrothricidae, Chydoridae, Bosminidae, Daphnidae, Ilyocryptidae, Moinidae y Sididae) siendo las dos primeras las mejor representadas $(60 \%$, y $35 \%$ del total respectivamente), mientras que en invierno Chydoridae representó $93 \%$ del total de cladóceros, y Macrothricidae solo el $4 \%$. La diversidad, la amplitud del nicho y el número de ítems presa fueron mayores en invierno. La superposición de la dieta entre los ejemplares de ambas estaciones fue alta $(\mathrm{C} \lambda=0.95)$; sin embargo, estos resultados podrían variar si la precisión en la identificación taxonómica aumentara. 
La dieta de $P$. australis mostró una mayor variación entre estaciones, con predominio de cladóceros en verano y quironómidos en el invierno (Fig. 3). El solapamiento de la dieta de los individuos entre estaciones fue bajo $(C \lambda=$ 0.51). En verano se registraron todos los ítems presa, y tanto la diversidad, como la amplitud del nicho fueron levemente mayores en esta estación (Cuadro 2).

Al analizar el solapamiento entre ejemplares de C. rachovii y $P$. australis recolectados en la misma estación, se encontró una mayor superposición en la dieta de individuos capturados en verano $(\mathrm{C} \lambda=0.85)$, que en aquellos recolectados en invierno $(\mathrm{C} \lambda=0.60)$. Las diferencias entre ambas especies fueron altamente significativas $(\chi 2, \mathrm{p}<0.0001)$ en las dos estaciones.

\section{DISCUSIÓN}

En las lagunas estudiadas, C. rachovii y $P$. australis son especies carnívoras, ambas se alimentan preferentemente de microcrustáceos y de larvas de dípteros y poseen variaciones en la dieta según la talla. Con base en los gremios tróficos propuestos por Ruiz Díaz (2005), ambas especies pertenecerían al grupo de consumidores de pequeños invertebrados.

En relación a la dieta de $C$. rachovii, nuestros resultados son similares a los reportados por Casciotta et al. (2005) para lagunas del Iberá, a los de Bonetto et al. (1978b) para lagunas de la cuenca del Riachuelo y a los de Teixeira de Mello et al. (2011) para ambientes de la República Oriental del Uruguay. Otros autores que trabajaron en ambientes loticos, señalan que $C$. rachovii tiene una dieta omnívora (Fernández et al., 2012) o insectívora en riachos del sur de Brasil (Bastos et al., 2013). Otras especies del género Characidium también se alimentan preferentemente de insectos (Barreto \& Aranha, 2006; Román-Valencia, Hernández, \& Samudio, 2007; Ferreira-Rezende, Mazzoni, Pellegrini-Caramaschi, Rodrigues, \& Moraes, 2011; Mazzoni et al., 2012).

Los resultados de este trabajo coinciden con los de Meschiatti y Arcifa (2002), Arias y Rossi (2005) y Casciotta et al. (2005) que indican una dieta carnívora para $P$. australis en ambientes lénticos, siendo los microcrustáceos e insectos sus principales componentes. En cambio Bonetto et al. (1978b) sugieren una dieta herbívora para esta especie.

La dieta para ambas especies estudiadas, muestra diferencias al variar la talla. Abelha et al. (2001) encuentran que la alimentación de una especie varía en las distintas etapas de su desarrollo, debido a diferencias en su demanda de energía y sus limitaciones morfológicas. De acuerdo a Houde (1997), a medida que los peces crecen, la variedad de presas consumidas se amplía; ya que la ingesta de alimento está limitada por la apertura de la boca del pez (Wootton, 1999). En relación a esto, P. australis mostró una clara tendencia al aumento en la variedad de presas y diversidad de la dieta en relación al tamaño de los individuos estudiados. En esta especie, fueron hallados un mayor porcentaje de microcrustáceos en ejemplares pequeños y un mayor número de insectos terrestres en individuos de mayor tamaño, resultados que coinciden con los de Meschiatti y Arcifa (2002) para esta especie.

En la dieta de C. rachovii, los ejemplares de las tres tallas mostraron una marcada preferencia por los microcrustáceos, y solo se observan diferencias significativas entre individuos de las tallas I y III. En contraposición a lo mencionado por Houde (1997), Barreto y Aranha (2006) encuentran una mayor amplitud del nicho trófico en individuos jóvenes de Characidium lalei. Ferriz, Fernández, López, $\&$ Bentos (2012) en un arroyo del centro de Argentina, no encuentran diferencias significativas entre tallas en la dieta de otra especie de Characidae (Pseudocorynopoma doriai).

Nuestros resultados indican que la dieta de ambas especies estudiadas varía estacionalmente. En C. rachovii los cambios más importantes fueron a nivel de recambio de las familias del ítem dominante (Cladocera) mientras que en la dieta de $P$. australis se registraron en verano casi el doble de ítem-presa que en invierno. Los cambios temporales en la dieta de los peces son muy comunes, y en general asociados con los 
periodos reproductivos de la presa (Wootton, 1999), las condiciones abióticas del ecosistema y la oferta de alimentos (Abelha et al., 2001). En grandes ríos y su planicie de inundación se han registrado diferencias en la dieta de peces entre periodos secos y húmedos, en relación a las fluctuaciones hidrológicas que determinan cambios en la oferta de alimentos (Winemiller et al., 2008).

Al comparar la dieta entre especies, el índice de Morisita reveló un importante solapamiento en la dieta de ejemplares de talla I, comprobando que comparten una elevada gama de recursos, siendo los cladóceros la presa más consumida. Machado-Allison (2005) reporta en áreas tropicales, un solapamiento en la dieta de especies de peces simpátricas, principalmente en las primeras etapas del desarrollo. Si bien en este estudio no se realizó un análisis de la oferta alimentaria, Cetra et al. (2011) y Ferreira-Rezende et al. (2011) afirman que los peces se alimentan de los recursos más abundantes y frecuentes del ambiente. Los cladóceros son habitantes frecuentes y abundantes en los ambientes acuáticos del Litoral Fluvial Argentino, sus poblaciones alcanzan altas densidades y constituyen una parte importante de la biomasa de comunidades como el plancton, el bentos y los complejos ligados a la vegetación (Paggi, 2004). Las larvas de quironómidos y los quidóridos (Cladocera) son muy abundantes en la vegetación acuática de una de las lagunas estudiadas (Poi de Neiff et al., 1999). Estudios realizados por Frutos, Poi de Neiff, \& Neiff (2009) en la laguna Soto, reportan un activo consumo de cladóceros por otros peces de tamaño y hábitos similar a los aquí estudiados.

La superposición de la dieta de ambas especies fue baja entre individuos de talla III, debido a un considerable aumento en el nicho trófico de $P$. australis, relacionado con la ingesta de insectos terrestres. Estos estuvieron entre los ítems de mayor volumen, motivo por el cual fueron poco frecuentes en ejemplares pequeños, ya que la ingesta de presas está limitada por la apertura de la boca del pez (Wootton, 1999). Arias y Rossi (2005) y Casciotta et al. (2005) también reportan el consumo de organismos alóctonos en $P$. australis, y lo relacionan con caracteres morfológicos que facilitarían la captura de presas en la interfase agua-aire, como ser la posición de la boca súpera, la cabeza aplanada y el perfil predorsal recto. De acuerdo a Machado-Allison (2005), muchos carácidos incluyen zooplancton inicialmente, cambiando a una dieta insectívora en adultos, con importante dependencia del material alóctono.

A pesar de la alta superposición de la dieta entre ambas especies, tanto el número de ítemspresa, como la diversidad y la amplitud del nicho trófico, indicaron que $P$. australis utilizó un amplio espectro trófico e incorporó elementos alóctonos, especialmente en los ejemplares de mayor tamaño. En cambio, C. rachovii mostró una tendencia hacia la especialización en la consumición de microcrustáceos. Bastos et al. (2013) caracteriza a C. rachovii como especialista, debido a la preferencia de la especie por unos pocos ítems, a pesar de consumir una gran variedad de presas raras, como parte de una estrategia a la vez oportunista. Nuestros resultados coinciden con los de Bastos et al. (2013), ya que en C. rachovii, a pesar de haberse encontrado 23 ítems alimenticos, la gran parte de estos tuvieron una baja abundancia y frecuencia, categorizándose como raros o accidentales, mientras que unas pocas presas (microcrustáceos y quironómidos) fueron las preferidas en su dieta. En lo que respecta a $P$. australis, nuestros resultados se corresponden con los de Arias y Rossi (2005) quienes califican a la especie como generalista, con especialización sobre cladóceros e insectos.

En ambas especies, la dieta de los individuos de tallas mayores fue más variada que en los más pequeños, motivo por el cual se acepta la hipótesis 1 . La hipótesis 2 , referida a que la alimentación fue más diversa durante el verano es aceptada para $P$. australis y no para $C$. rachovii. La hipótesis 3 es rechazada parcialmente porque ambas especies tuvieron un elevado solapamiento de nichos en sus primeros estadios, el cual disminuyó en los ejemplares de mayor tamaño.

Las lagunas estudiadas están sujetas a una creciente urbanización en sus márgenes, 
motivo por el cual si el recurso trófico predominante en el ambiente disminuye por aumento de la acción antrópica, las especies en estudio podrían competir por el alimento dado el elevado solapamiento de su nicho trófico.

\section{AGRADECIMIENTOS}

Agradecemos a Juan José Neiff por sus enseñanzas y aportes durante el desarrollo de este trabajo, a Marta Canon Verón por su ayuda en la disección de peces y su colaboración en la determinación de los mismos, a Santa Margarita Frutos por su enseñanza en la determinación de microcrustáceos, a Miryam Damborsky por sus correcciones en el manuscrito, y a la editora y los tres revisores anónimos por sus comentarios y sugerencias, que ayudaron a mejorar significativamente el manuscrito. El proyecto Análisis del estado ecológico de lagunas periurbanas (Corrientes, Argentina) PI 2011Q001 SGCYT-UNNE financió el estudio.

\section{RESUMEN}

Los peces exhiben diversas estrategias de alimentación que pueden sufrir modificaciones a través de la ontogenia o estacionalmente con implicaciones ecológicas en las cadenas tróficas. Aún cuando se reconoce la importancia de los peces como depredadores tope, la dieta de muchos de ellos es todavía escasamente conocida, especialmente de aquellos que habitan las lagunas someras densamente cubiertas de vegetación. El objetivo de este estudio fue investigar la dieta de Characidium rachovii y Pyrrhulina australis de diferentes clases de tamaños (I, II y III) durante el invierno y el verano y estimar la amplitud de su nicho trófico. El trabajo de campo se llevó a cabo en dos lagos someros alimentados por lluvias (Lagunas Soto y Pampin, provincia de Corrientes) entre julio 2011 y agosto 2012. Se examinaron los contenidos estomacales de 104 individuos de C. rachovii y de 91 especímenes de $P$. australis. La curva de acumulación indicó que el número de estómagos analizados fue representativo para ambas especies. En el contenido estomacal se reconocieron 23 ítems alimenticios en $C$. rachovii y 28 en $P$. australis. Ambas especies consumieron preferentemente cladóceros y larvas de quironómidos según el índice de importancia relativa (IRI) y mostraron un elevado solapamiento en sus dietas $(85 \%)$ de acuerdo al índice de Morisita. Sin embargo, la diversidad de ítems y la amplitud del nicho trófico fue mayor en $P$. australis $\left({ }^{I} D=8.86 ; B=4.76\right)$ respecto de C. rachovii $\left({ }^{1} D=3.37 ; B=2.25\right)$. Se registraron diferencias significativas en la dieta de las tres tallas consideradas para P. australis $(\chi 2, \mathrm{p}<0.01)$ y entre las tallas I y III de $C$. rachovii $(\chi 2, p=0.03)$. En ambas especies la diversidad de ítems alimenticios aumentó con el incremento de sus tallas. Las diferencias en la dieta entre estaciones fueron significativas para las dos especies $(\chi 2, \mathrm{p}<0.0001)$. La alta superposición en las dietas, indica que ambas especies utilizan predominantemente los mismos recursos. A pesar de esto, tanto el número de ítems presa como la diversidad y la amplitud del nicho trófico, indican que estas especies utilizan estrategias distintas, ya que $C$. rachovii mostró una tendencia a la especialización en el consumo de microcrustáceos; mientras que $P$. australis exhibió un espectro trófico más amplio, incorporando insectos de áreas litorales y de la comunidad terrestre. Las lagunas estudiadas están sujetas a una creciente urbanización en sus márgenes, motivo por el cual si el recurso trófico predominante en el ambiente disminuye por aumento de la acción antrópica, las especies en estudio podrían competir por el alimento dado el elevado solapamiento de su nicho trófico.

Palabras clave: peces, dieta, nicho trófico, lagunas periurbanas, noreste argentino.

\section{REFERENCIAS}

Abelha, M. C., Agostinho, A. A., \& Goulart, E. (2001). Plasticidade trófica em peixes de água doce. Acta Scientiarum. Biological Sciences, 23, 425-434.

Arias, J. \& Rossi, L. (2005). Dieta y estrategia alimentaria de Pyrrhulina australe (Pisces, Lebiasinidae). Revista FABICIB, 9, 197-205.

Barreto, A. P. \& Aranha, J. M. R. (2006). Alimentação de quatro espécies de Characiformes de um riacho da Floresta Atlântica, Guaraqueçaba, Paraná, Brasil. Revista Brasileira de Zoologia, 23, 779-788.

Bastos, R. F., Miranda, S. F., \& Garcia, A. M. (2013). Dieta e estratégia alimentar de Characidium rachovii (Characiformes, Crenuchidae) em riachos de planície costeira do sul do Brasil. Iheringia, Série Zoologia, Porto Alegre, 103, 335-341.

Bonetto, A. A., Neiff, J. J., Poi de Neiff, A., Varela, M. E., Corrales, M. A., \& Zalocar, Y. (1978a). Estudios limnológicos en la cuenca del Riachuelo. III: Laguna Brava. Ecosur, 5, 57-84.

Bonetto, A. A., Roldán, D., \& Oliver, M. E. (1978b). Estudios limnológicos en la cuenca del Riachuelo (Corrientes, Argentina). I: Poblaciones de peces en ambientes leníticos y lóticos. Ecosur, 5, 1-15.

Bruniard, E. D. (1999). Los regímenes hídricos de las formaciones vegetales. Aportes para un modelo fotoclimático mundial. Resistencia, Chaco: Editorial Universitaria del Nordeste. 
Casciotta, J., Almirón, A., \& Bechara, J. (2005). Peces del Iberá - Hábitat y Diversidad. La Plata, Argentina: Editorial Grafikar.

Cetra, M., Rodrigues-Rondineli, G., \& Pereira-Souza, U. (2011). Compartilhamento de recursos por duas espécies de peixes nectobentônicas de riachos na bacia do rio Cachoeira (BA). Biota Neotropica, 11, 87-95.

Colwell, R. K. (2006). EstimateS: statistical estimation of species richness and shared species from samples (software and user's guide). Versión 8.0. Recuperado de http://purl.oclc.org/estimates

Costalago, D., Navarro, J., Álvarez-Calleja, I., \& Palomera I. (2012). Ontogenetic and seasonal changes in the feeding habits and trophic levels of two small pelagic fish species. Marine Ecology Progress Series, 460, 169-181.

Dunham, A. E. (1983). Realized niche overlap, resource abundance, and intensity of interespecific competition. In R. B. Huey, E. R. Pianka, \& T. W. Schoener (Eds.), Lizard Ecology (pp. 261-280). Cambridge, Massachusets: Harvard University Press.

Fernández, E. M., Ferriz, R. A., Bentos, C. A., \& López, G. R. (2012). Dieta y ecomorfología de la ictiofauna del arroyo Manantiales, provincia de Buenos Aires, Argentina. Revista del Museo Argentino de Ciencias Naturales, 14, 1-13.

Fernández, H. R. \& Domínguez, E. (2009). Macroinvertebrados bentónicos sudamericanos. Sistemática y Biología. Tucumán, Argentina: Universidad Nacional de Tucumán.

Ferreira-Rezende, C., Mazzoni, R., Pellegrini-Caramaschi, É., Rodrigues, D., \& Moraes, M. (2011). Prey selection by two benthic fish species in a Mato Grosso stream, Rio de Janeiro, Brazil. Revista de Biología Tropical, 59, 1697-1706.

Ferriz, R. A., Fernández, E. M., López, G. R., \& Bentos, C. A. (2012). Alimentación de Pseudocorynopoma doriai (Pisces: Characidae) en el arroyo El Portugués, provincia de Buenos Aires, Argentina. Revista del Museo Argentino de Ciencias Naturales, 14, 243-251.

Frutos, S. M., Poi de Neiff, A. S. G., \& Neiff, J. J. (2009). Zooplankton abundance and species diversity in two lakes with different trophic states (Corrientes, Argentina). Acta Limnologica Brasiliensia, 21, 367-375.

George, E. L., \& Hadley, W. F. (1979). Food and habitat partitioning between rock bass (Ambloptides rupestris) and smallmouth bass (Micropterus dolomieu) young of the year. Transaction of American Fisheries Society, 108, 253-257.

Horn, H. S. (1966). Measurement of "overlap" in comparative ecological studies. The American Naturalist, $100,419-424$
Houde, E. D. (1997). Patterns and consequences of selective processes in teleost early life histories. In C. Chambers \& E. A. Trippel (Eds.), Early life history and recruitment in fish populations (pp. 173-196). London: Chapman \& Hall.

Hurlbert, S. H. (1978). The measurement of niche overlap and some relatives. Ecology, 59, 67-77.

INTA. (2009). Zonas AgroEconómicas Homogéneas Corrientes. En F. Acosta, L. Giménez, C. Calvi, \& M. Richieri (Eds.), Estudios socioeconómicos de la sustentabilidad de los sistemas de producción y recursos naturales (pp. 1-75). Buenos Aires: INTA.

Jost, L. (2006). Entropy and diversity. Oikos, 113, 363-375.

Levins, R. (1968). Evolution in changing environments: some theoretical explorations. New Jersey: Princeton University Press.

Lopez, H. L., Miquelarena, A. M., \& Ponte Gómez, J. (2005). Biodiversidad y Distribución de la Ictiofauna Mesopotámica. INSUGEO, Miscelánea, 14, 311-354.

Lopretto, E. \& Tell, G. (1995). Ecosistemas de aguas continentales. Metodologías para su estudio. La Plata, Argentina: Ediciones Sur.

Machado-Allison, A. (2005). Los peces de los llanos de Venezuela: un ensayo sobre su historia natural. Caracas, Venezuela: Consejo de Desarrollo Científico y Humanístico.

Mazzoni, R., Marques, P. S., Rezende, C. F., \& IglesiasRios, R. (2012). Niche enlargement as a consequence of co-existence: a case study. Brazilian Journal of Biology, 72, 267-274.

Meschiatti, A. J., \& Arcifa, M. S. (2002). Early life stages of fish and the relationships with zooplankton in a tropical brazilian reservoir: Lake Monte Alegre. Brazilian Journal of Biology, 62, 41-50.

Montori, A. (1991). Alimentación de los adultos de Euproctus asper (Duges 1852) en la montaña media del Prepirineo catalán (España). Revista Española de Herpetología, 5, 23-36.

Neiff, J. J. (2004). El Iberá... ¿en peligro?. Buenos Aires: Fundación Vida Silvestre Argentina.

Paggi, J. C. (2004). Importancia de la fauna de "Cladóceros " (Crustácea, Branchiopoda) del Litoral Fluvial Argentino. INSUGEO, Miscelánea, 12, 239-246.

Piet, G. J., Ret, J. S., Guruge, W. A., Vijuever, J., \& Van Densen, W. L. (1999). Resource partitioning along three niche dimentions in a size-structured tropical fish assemblege. Canadian Journal of Fisheris and Aquatic Sciences, 56, 1241-1254.

Poi, A. \& Galassi, M. E. (2013). Sistema 4b - Humedales del noroeste de Corrientes. En L. Benzaquen, D. 
Blanco, R. Bo, P. Kandus, G. Lingua, P. Minotti, \& L. Vidal (Eds.), Inventario de los humedales de Argentina: sistemas de paisajes de humedales del corredor fluvial Paraná Paraguay (pp. 215-222). Buenos Aires: Secretaría de Ambiente y Desarrollo Sustentable de la Nación.

Poi de Neiff, A. S., Neiff, J. J., Patiño, C. A., Ramos, A. O., Cáceres, J. R., Frutos, S. M., \& Verón, C. (1999). Estado trofico de dos lagunas en planicies anegables con areas urbanas de la provincia de Corrientes. FACENA, 15, 93-110.

TspDig. (Version 2.17.) [Computer software] Stony Brook, Department of Ecology and Evolution, State University of New York: Rohlf, J. F.

Román-Valencia, C., Hernández, J. H., \& Samudio, H. F. (2007). Sobre Ecología de Characidium caucanum (Pisces: Crenuchidae) en el alto río Cauca, Colombia. Asociación Colombiana de Ictiólogos- DAHLIA, 9, 33-42.

Ross, S. T. (1986). Resource partitioning in fish assemblages: A review of field studies. Copeia, 352-388.
Ruiz-Díaz, F. (2005). Alimentación. En J. Casciotta, A Almirón, \& J. Bechara (Eds.), Peces de Iberá. Hábitat y Diversidad (pp. 41-45). La Plata, Argentina: Editorial Grafikar.

Teixeira de Mello, F., González-Bergonzoni, I., \& Loureiro, M. (2011). Peces de agua dulce del Uruguay. Uruguay: PPR-MGAP.

Welcomme, R. L. (1992). Pesca Fluvial (Documento técnico de pesca - 262). Roma: FAO.

Winemiller, K. O. (1989). Ontogenetic diet shifts and resource partitioning among piscivorous fishes in the Venezuelan llanos. Environmental Biology of Fishes, 26, 177-199.

Winemiller, K. O., Agostinho, A. A., \& Caramaschi, E. P. (2008). Fish Ecology in Tropical Streams. In D. Dudgeon. (Ed.), Tropical Stream Ecology (pp. 107-146). Amsterdam: Elsevier.

Wootton, R. J. (1999). Ecology of teleost fishes. The Netherlands: Kluwer Academic Publishers. 
